\title{
Cluster scattering effects on phonon conduction in graphene
}

\author{
Natalio Mingo, ${ }^{1,2}$ K. Esfarjani, ${ }^{2}$ D. A. Broido, ${ }^{3}$ and D. A. Stewart ${ }^{4}$ \\ ${ }^{1}$ LITEN, CEA-Grenoble, 17 rue des Martyrs, 38054 Grenoble Cedex 9, France \\ ${ }^{2}$ The University of California at Santa Cruz, Santa Cruz, California 95064, USA \\ ${ }^{3}$ Department of Physics, Boston College, Chestnut Hill, Massachusetts 02467, USA \\ ${ }^{4}$ Cornell Nanoscale Facility, Cornell University, Ithaca, New York 14853, USA
}

(Received 1 July 2009; revised manuscript received 20 October 2009; published 13 January 2010)

\begin{abstract}
The phonon-scattering cross section associated with isotopic clusters is evaluated from first principles and used to estimate the reduction in thermal conductance of wide graphene samples. A strong sensitivity of the thermal conductivity toward clustering is predicted for micrometer-sized samples at low temperatures. Important differences are obtained between the atomistically computed cross section, and existing analytical approximations, emphasizing the importance of atomistic investigations of thermal transport. Finally, possible techniques are suggested for synthesizing graphene containing isotopic clusters.
\end{abstract}

DOI: 10.1103/PhysRevB.81.045408

PACS number(s): 61.48.De, 63.22.-m, 63.20.kp, 65.80.-g

\section{INTRODUCTION}

It is a known fact that impurity aggregates may scatter low-frequency phonons much more efficiently than if they were acting separately. ${ }^{1-3}$ This has been recently used to develop thermoelectric materials of the "nanoparticle in alloy" type, whereby the addition of nanoparticles reduces the alloy's thermal conductivity dramatically. ${ }^{4,5}$ Although the basic explanation is simple, ${ }^{5}$ accurate computations of the scattering cross section of the nanoparticles are lacking. Reference 6 proposed an interpolation approach to link between the Rayleigh and geometric scattering limits associated with the low and high frequencies, respectively. Reference $7 \mathrm{im}$ proved on that crude model via Mie's theory of scattering but it still relied on the elastic medium approximation. It is clear that further progress in the theoretical investigation of materials' thermal conduction requires a fully atomistic approach free of the simplifying assumptions employed to date. In confined systems such as nanotubes, nanoribbons, or thin nanowires, the Green's-function approach presented in Refs. $8-10$ is well suited. However, for extended two-dimensional (2D) or three-dimensional systems, such an approach is not feasible since the size of the slabs becomes intractable.

To overcome the issues above, here we present a general formalism to compute the total scattering cross section due to an arbitrary scatterer in an extended material, in a parameter free, atomistic ab initio scheme. The method is illustrated for the case of isotope scattering in graphene, a material of strong interest nowadays due to its conceptual simplicity and promising fields of application. The calculated cross sections have a direct application in the evaluation of graphene's thermal conductivity, which is found to be strongly sensitive to the size of the isotope cluster. At the end of the paper we propose a possible method to synthesize graphene containing isotopic clusters, which might allow to experimentally verify the effects predicted.

\section{ATOMISTIC COMPUTATION OF SCATTERING CROSS SECTIONS}

The goal of this section is to introduce a compact formulation to compute the scattering cross section in a local basis representation. This formulation is applicable both to phonon scattering and to tight-binding electron scattering. In this paper we only apply it to phonon scattering. The equations can be directly derived for phonons, without the need to pass through the electron formulation, using techniques such as those in Ref. 11. Nonetheless, we find it simpler to make the general derivation for the electronic case first and afterward convert the equations to the phonon case.

The total scattering cross section in the electron-scattering case can be expressed as ${ }^{12}$

$$
\sigma=\frac{\Omega}{d E / d k} 2 \pi \sum_{\mathbf{k}_{\mathbf{f}}}\left|\left\langle\mathbf{k}_{f}\left|T^{+}(E)\right| \mathbf{k}\right\rangle\right|^{2} \delta\left(E_{f}-E\right),
$$

where $|\mathbf{k}\rangle$ is the incident eigenstate and $\left|\mathbf{k}_{f}\right\rangle$ are the propagating eigenstates of the unperturbed Hamiltonian. $\Omega$ is the volume into which the wave functions are normalized. The $\mathrm{T}$ matrix is defined as

$$
T^{+(-)}=\mathbf{V}+\mathbf{V G}^{+(-)} \mathbf{V},
$$

where $\mathbf{V}$ is the perturbation operator and $\mathbf{G}$ is the perturbed Green's function. ${ }^{12}$ The total-cross-section expression can be transformed into a more practical one. It suffices to use the following identity:

$$
\begin{aligned}
& \mathbf{T}^{+}-\mathbf{T}^{-}=\mathbf{V}\left(\mathbf{G}^{+}-\mathbf{G}^{-}\right) \mathbf{V} \\
&=-2 \pi i \mathbf{V}\left[\sum_{m} \delta\left(E_{m}-E\right)\left|\Psi_{m}\right\rangle\left\langle\Psi_{m}\right|\right] \mathbf{V} \\
&=-2 \pi i \mathbf{V}\left[\sum_{m} \delta\left(E_{m}-E\right)\left(\mathbf{I}+\mathbf{g}^{+} \mathbf{T}^{+}\right)\left|\phi_{\mathbf{m}}\right\rangle\left\langle\phi_{\mathbf{m}}\right|\left(\mathbf{I}+\mathbf{T}^{-} \mathbf{g}^{-}\right)\right] \mathbf{V},
\end{aligned}
$$

where $\mathbf{g}$ is the unperturbed Green's function, $\phi$ are the unperturbed eigenfunctions, and $\Psi$ are the eigenfunctions of the full Hamiltonian. The above can be recasted as

$$
\mathbf{T}^{+}-\mathbf{T}^{-}=-2 \pi i \sum_{m} \mathbf{T}^{+}\left|\phi_{m}\right\rangle \delta\left(E_{m}-E\right)\left\langle\phi_{m}\right| \mathbf{T}^{-},
$$

where we have used 


$$
T^{+(-)}=\left(\mathbf{I}+\mathbf{T}^{+(-)} \mathbf{g}^{+(-)}\right) \mathbf{V}=\mathbf{V}\left(\mathbf{I}+\mathbf{g}^{+(-)} \mathbf{T}^{+(-)}\right) .
$$

Using this with Eq. (1) it is trivial to show that

$$
\sigma=-\frac{2 \Omega}{d E / d k} \operatorname{Im}\left\{\left\langle\mathbf{k}\left|T^{+}(E)\right| \mathbf{k}\right\rangle\right\} .
$$

This is just the well-known "optical theorem," expressed in a matrix form ready to be evaluated.

It is now straightforward to translate the obtained expressions to the scattering cross section of lattice waves or phonons. Single-particle electron and phonon stationary problems are completely equivalent, if one makes the substitutions: $E \rightarrow \omega^{2}, \mathbf{H} \rightarrow \mathbf{K}$, where $\omega$ is the phonon frequency and $\mathbf{K}$ is the mass normalized force constants (FCs) matrix, ${ }^{13}$

$$
K_{i j}=\frac{1}{\sqrt{M_{i} M_{j}}} \frac{\partial^{2} U}{\partial u_{i} \partial u_{j}},
$$

where $U$ is the energy of the system, $u_{i}$ is the displacement of the $i$ degree of freedom, and $M_{i}$ is the mass of the atom to which this degree of freedom belongs. Thus for phonons

$$
\begin{aligned}
\sigma & =\frac{\Omega}{2 \omega v_{g}} 2 \pi \sum_{\mathbf{k}_{\mathbf{f}}}\left|\left\langle\mathbf{k}_{f}\left|T^{+}\left(\omega^{2}\right)\right| \mathbf{k}\right\rangle\right|^{2} \delta\left(\omega_{f}^{2}-\omega^{2}\right) \\
& =-\frac{2 \Omega}{2 \omega v_{g}} \operatorname{Im}\left\{\left\langle\mathbf{k}\left|T^{+}\left(\omega^{2}\right)\right| \mathbf{k}\right\rangle\right\} .
\end{aligned}
$$

where $v_{g}$ is the group velocity of the incident phonon.

\section{FIRST-PRINCIPLES CALCULATION OF FORCE CONSTANTS}

The force constants were extracted from first-principles calculations. The VASP (Ref. 14) code was used to perform a series of force-displacement calculations in a supercell of 48 atoms. We employed the projector augmented wave method within the generalized gradient approximation (PAW-GGA) with PW91 functional, with a $430 \mathrm{eV}$ cutoff and $13 k$ points within the irreducible zone. The range of the force constants was restricted to the fourth neighbor. Since translational and rotational invariances needed to be enforced in order to get a physically sound set of force constants, small deviations were obtained from the true value of the FCs obtained by doing a finite difference calculation. The methodology to extract the FCs is explained in detail in a previous paper. ${ }^{15}$ It basically reduces all the FCs present in the Taylor expansion of the potential energy up to second order, by using the symmetry and invariance constraints to a few number of independent FCs. The latter are extracted from the computed set of force-displacement data from first principles, by using a singular value decomposition.

We show the phonon dispersion and density of states in Fig. 1. The results including up to four or up to ten nearest neighbors are plotted together for comparison. The phonondispersion curve for four nearest neighbors is already in good agreement with the calculations including higher number of neighbors. The dispersion compares well with other theoretical calculations ${ }^{17}$ and with experimental measurements on graphite. ${ }^{16}$ We note that the weak coupling between layers in

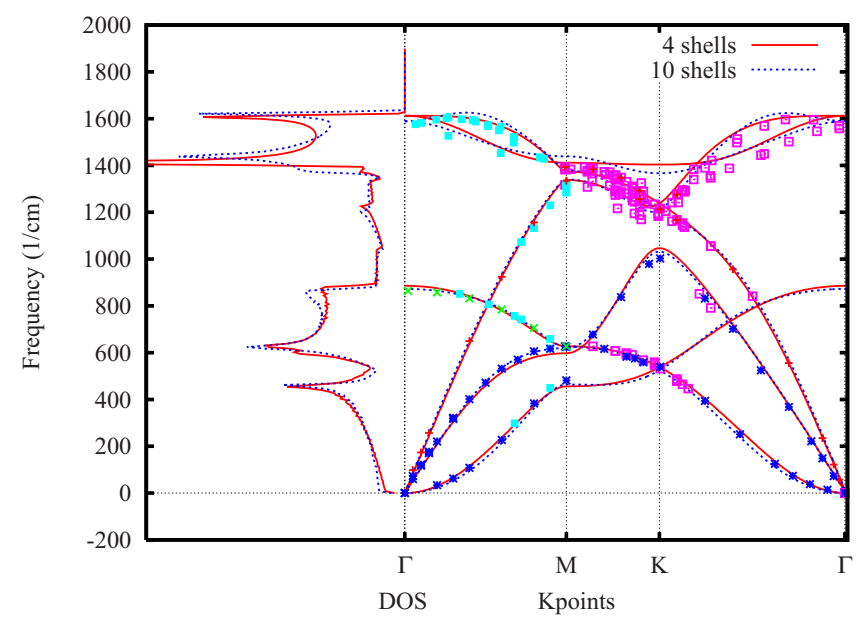

FIG. 1. (Color online) Phonon dispersion and density of states obtained from the $a b$ initio force constants keeping interactions to four and ten nearest neighbors. Experimental measurements on graphite from Ref. 16 are also shown for comparison.

graphite makes graphene and graphite in-plane dispersions almost identical. $^{18}$

\section{NUMERICAL EVALUATION OF HARMONIC GREEN'S FUNCTIONS}

The total cross section has then been evaluated in the harmonic approximation using the approach described above, for isotope clusters of different sizes (Fig. 2.) The most demanding computational step is the computation of the perfect crystal's harmonic Green's-function matrix, $\mathbf{g}\left(\omega^{2}\right)$, in a region of real space large enough to contain the impurity cluster. There are different ways of doing this. The most straightforward option is based on the root-sampling method. ${ }^{11}$ In this approach one first numerically computes the phonon spectral density from the solution of the eigenvalue problem in q space, with Born-Von Karman boundary conditions in the two directions parallel to the infinite graphene sheet. This yields the imaginary part of the Green's function. Then one can use a Hilbert transformation to obtain the Green's-function's real part. ${ }^{19}$ The disadvantage of this approach is the very large number of $\mathbf{q}$ points one needs to

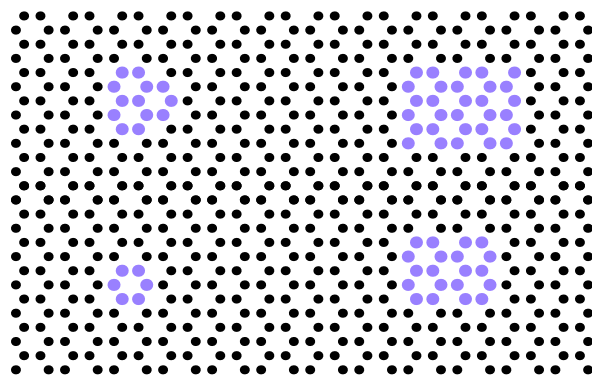

FIG. 2. (Color online) Clusters with 6, 13, 20, and $30{ }^{13} \mathrm{C}$ isotopes (in blue), employed in the calculations. The abscissa and ordinate axes, respectively, correspond to the $z$ and $x$ directions referred to in the text 
consider in the Brillouin zone, in order to obtain accurate results.

An alternative to this reciprocal-space approach is the recursion method, ${ }^{20}$ which obtains the Green's function directly in real space. This method is very well suited to disordered systems but it is not advantageous when dealing with periodic systems. It is thus less efficient than the technique we proceed to describe below.

Instead of the purely reciprocal-space root-sampling technique, or the purely real-space recursion method, we employed a hybrid computation scheme, making use of a wellknown renormalization technique. This method of computing the Green's function has been described in depth in Refs. 21 and 22, and we refer to those publications for additional details. The approach uses a hybrid reciprocal-real space representation for the system's degrees of freedom. Considering the graphene sheet to be laid parallel to the $(x, z)$ plane, we employ the real-space representation along the $z$ direction but we use a reciprocal-space representation with a Born-Von Karman boundary condition along the $x$ direction. This permits us to split the system into a set of independent onedimensional (1D) systems, each of them corresponding to a different transverse wave vector $q_{x}$. The nonzero forceconstant matrices are then denoted as $\mathbf{K}_{0,0}\left(q_{x}\right)$, for the matrix connecting elements within the $1 \mathrm{D}$ unit cell, and $\mathbf{K}_{0,1}\left(q_{x}\right)$ and $\mathbf{K}_{0,-1}\left(q_{x}\right)$, for the matrices connecting the 1D unit cell to its nearest cell on either side. The unit cell is chosen large enough so that no interactions exist between nonneighboring cells. In the four nearest-neighbor case considered, this corresponds to a four-atom unit cell, i.e., the matrices are $12 \times 12$. In this representation, we can obtain the independent Green's functions $\mathbf{g}_{m, n}\left(q_{x}, \omega^{2}\right)$, where $m, n$ denote different unit cells along the $z$ direction. The real-space Green's function between two arbitrary atomic degrees of freedom $k$ and $l$ in the system, can be obtained as $g_{k, l}$ $=\sum_{q_{x}} e^{i q_{x}\left(X_{k}-X_{l}\right)} g_{m(k), n(l)}$, where $m(k)$ and $n(l)$ are the corresponding unit cells along the $z$ direction associated with degrees of freedom $k$ and $l$, respectively, and $X_{k}$ and $X_{l}$ are the $x$ coordinates of the atoms to which $k$ and $l$ belong.

To compute the $\mathbf{g}_{m, n}\left(q_{x}, \omega^{2}\right)$ we proceed as follows. First, we determine the size of the area that will fully contain the impurity cluster afterward. The largest area we considered consisted of $6 \times 7$ unit cells, each of them containing four atoms. The length of this area in the $z$ direction is that of six unit cells. The force constants matrix associated to this central region is constructed in the hybrid representation, $\mathbf{K}^{\mathbf{c}}\left(q_{x}\right)$. The dimension of this matrix is six times larger than that of $\mathbf{K}_{0,0}\left(q_{x}\right)(72 \times 72)$. Then, the self-energies $\mathbf{\Sigma}_{-1}\left(q_{x}, \omega\right)$ and $\Sigma_{1}\left(q_{x}, \omega\right)$ acting on this central region are computed using the decimation technique of Ref. 23. This renormalization method consists in successively doubling the size of a cluster, to compute the local Green's functions and selfenergies of a semi-infinite system with only a small number of iterations. We used 24 iterations, which yields a system of macroscopic size in the $z$ direction. The two self-energies corresponding to the two sides (labeled 1 and -1) of the central part are then added to the mass normalized force constants matrix, and the Green's function is computed as

$$
\mathbf{g}_{m, n}\left(q_{x}, \omega^{2}\right)=\left[\omega^{2} \mathbf{I}-\mathbf{K}^{\mathbf{c}}\left(q_{x}\right)-\Sigma_{-1}\left(q_{x}, \omega^{2}\right)-\Sigma_{1}\left(q_{x}, \omega^{2}\right)\right]^{-1} .
$$

In this equation, $\omega^{2}$ is a real quantity since the self-energies already include the necessary imaginary component. The standard small imaginary part of the frequency is only introduced in the decimation algorithm when computing the selfenergy, where $\omega^{2}$ needs to be replaced by $\omega^{2}+i \delta \omega$. In the computations presented we used $\delta=5 \mathrm{THz}$.

Since the $\mathbf{q}$ vectors are considered only along one of the dimensions, this approach requires a much smaller number of $q$ points, than if we did the full calculation in real space $(N$ points in the former case compared to $N^{2}$ points in the latter). In addition, our hybrid method yields the Green's function directly, without any need to Hilbert transform to obtain the real part. For these reasons, we find this approach to be more convenient than the approach based on Hilbert transforming the density of states obtained by root sampling. Although the cross section is independent of the method used to compute the Green's function, it would be interesting to compare results using the latter approach. Nevertheless, such study falls out of the scope of the present paper.

We used a grid of $100 q_{x}$ points in the $x$ direction. After computing the perfect crystal's Green's-function matrix in the selected region, we introduce the perturbation induced by the scattering cluster on the force constants matrix. This perturbation matrix is defined in real space as the difference between the force constants in the system with the impurities, and the force constants of the perfect crystal, $\mathbf{V} \equiv \mathbf{K}^{\prime}$ $-\mathbf{K}_{0}$. Thus, $\mathbf{V}$ is nonzero only in a defined region of space around the impurity cluster. In the case of isotope impurities, we have

$$
V_{k, l}=\left(\sqrt{\frac{M_{k} M_{l}}{M_{k}^{\prime} M_{l}^{\prime}}}-1\right) K_{k, l},
$$

where $M_{k}$ denotes the mass of the atom to which degree of freedom $k$ belongs.

We then compute the T matrix for each cluster configuration as

$$
\mathbf{T}\left(\omega^{2}\right)=\left[\mathbf{I}-\mathbf{V g}\left(\omega^{2}\right)\right]^{-1} \mathbf{V} .
$$

Finally the total cross section is obtained using Eq. (8).

\section{RESULTS}

\section{A. Cross sections}

The calculated total scattering cross sections for phonons along the $\Gamma-\mathrm{M}$ direction are shown in Fig. 3. It is clear from the figure that at low frequency the results match the analytical expressions for the 2D Rayleigh scattering of an elastic medium. The quadratic out of plane mode is completely decoupled from the linear modes, and adding any arbitrary distribution of isotopic impurities does not change this [this is evident from Eq. (10)]. Thus, for the Rayleigh cross sections of a cluster containing $N$ atoms one analytically obtains

$$
\sigma_{\text {linear }}=\frac{1}{4} S_{0}^{2}\left(N \frac{\Delta M}{M}\right)^{2} \omega^{3} / c^{3},
$$




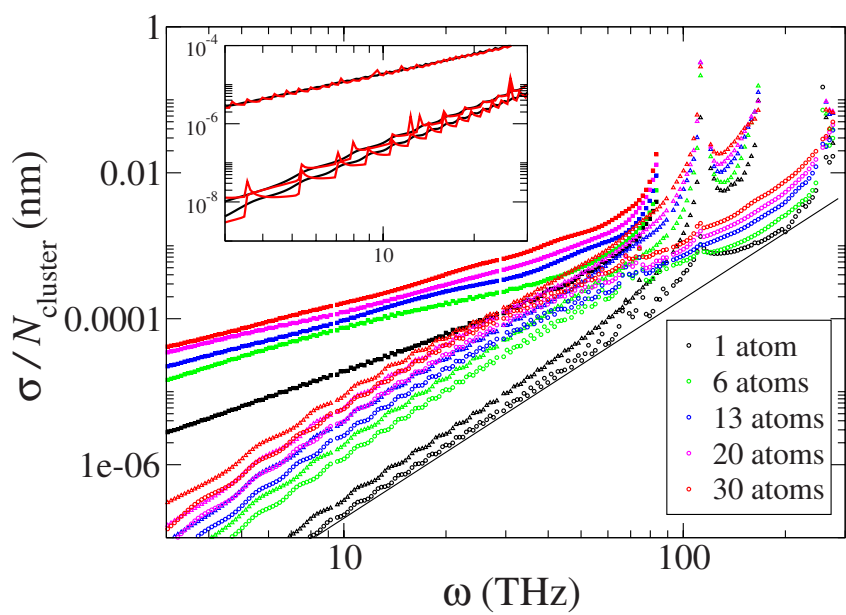

FIG. 3. (Color online) Total scattering cross section divided by number of atoms in the cluster, versus angular frequency, for 1,6 , 13,20 , and $30{ }^{13} \mathrm{C}$ isotope clusters. The largest, medium, and smallest cross section of each cluster correspond to the quadratic, transverse, and longitudinal phonon branch, respectively. Solid line: analytical expression, Eq. (12), for longitudinal branch with 1 isotope impurity. Inset: comparison between low-frequency results obtained with $\delta=0.005 \mathrm{THz}$ (red lines) and $\delta=5 \mathrm{THz}$ (black lines). Spurious singularities develop if the imaginary part of the frequency is taken too small.

$$
\sigma_{\text {quadratic }}=\frac{1}{16 \alpha^{3 / 2}} S_{0}^{2}\left(N \frac{\Delta M}{M}\right)^{2} \omega^{3 / 2},
$$

where $S_{0}$ is the surface area per atom, $c$ is the linear mode's speed of sound, and $\omega=\alpha q^{2}$ for the quadratic mode.

If the imaginary part of the frequency is too small, the atomistically computed cross section displays Van-Hovetype singularities at the lowest frequencies, due to the $k_{\|}$ mesh used. Choosing a large enough $\delta$ makes this artifact disappear. The inset of Fig. 3 shows that $\delta=5 \mathrm{THz}$ yields proper smooth results, although some small numerical undulations still remain at the lowest frequencies.

At high frequency the atomistic results markedly differ both from the geometric limit and from the Rayleigh analytical expression. This is especially clear for the singleimpurity case, which scatters considerably more strongly than what the analytical approximation would predict at high frequency.

As the cluster size is increased, the maximum frequency where Rayleigh expressions, Eqs. (12) and (13), are valid shifts to lower frequencies. The case of the quadratic branch is remarkable in this respect. Although at the lowest frequencies this branch behaves in the $N^{2}$ way predicted by the Rayleigh formula, it deviates considerably from this behavior already at frequencies as low as $10 \mathrm{THz}$ (see Fig. 4). A consequence of this is that the interpolation formula between low and high frequencies developed in Ref. $6, \sigma^{-1}$ $\simeq \sigma_{\text {Rayleigh }}^{-1}+\sigma_{\text {Geom }}^{-1}$ fails badly in reproducing the actual cross section for the quadratic branch. From Fig. 4 it is clear that the geometrical cross section is still much larger than the Rayleigh cross section, and therefore the approximated interpolation expression cannot account for the observed devia-

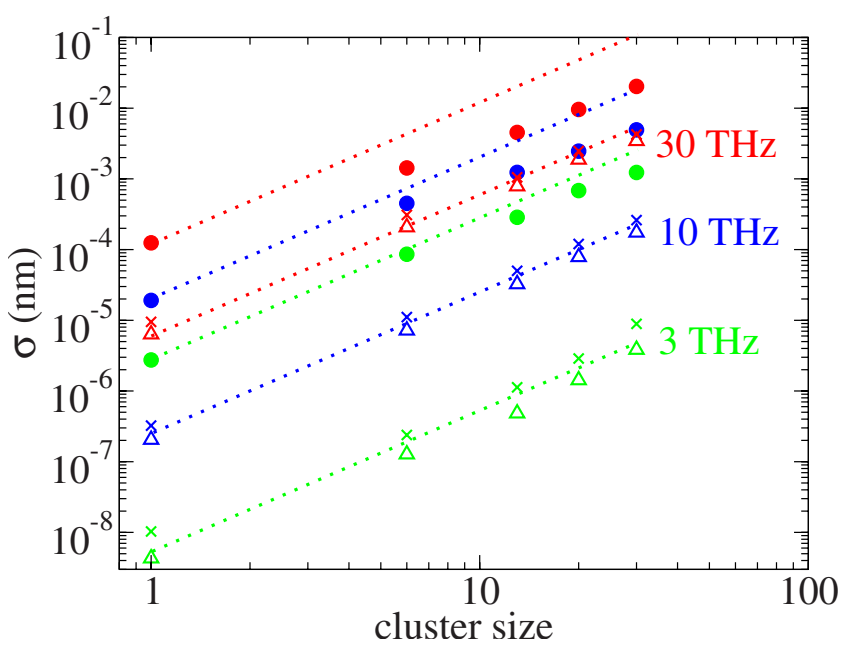

FIG. 4. (Color online) Total scattering cross section at selected frequencies of 3 (green), 10, (blue) and $30 \mathrm{THz}$ (red), plotted as a function of cluster size, for the three acoustic branches. Triangles: longitudinal branch; crosses: transverse branch; circles: quadratic out of plane branch. Whereas the two linear branches follow well the $N^{2}$ dependence predicted by the Rayleigh approximation, the quadratic branch clearly deviates from this dependence for the 10 and $30 \mathrm{THz}$ cases.

tion from the Rayleigh formula. All this underlines the importance of performing atomistic calculations.

\section{B. Effects on the thermal conductance}

For a random distribution of scatterers the phonon lifetime is directly related to the scatterer's total cross section by $\tau^{-1}(\omega)=v_{g}(\omega) \sigma(\omega) / A$, where $1 / A$ is the areal density of scatterers. Reference 4 directly applied this to evaluate the thermal conductivity of a nanocomposite, using the elastic medium cross sections given by the optical theorem, and the interpolating scheme to link with the geometric limit. Strictly speaking however, it is not correct to employ the total cross section when evaluating the bulk material's thermal conductivity. For transport involving one phonon branch, the proper solution to the Boltzmann equation yields the thermal conductivity in terms of the transport cross section, which coincides with the total cross section only if scattering is equiprobable in all scattered directions. ${ }^{3}$ In the presence of multiple branches, a full solution of the Boltzmann equation is required. In addition, anharmonic interactions turn the problem into an inelastic one, considerably increasing its computational difficulty. Localization may also be an issue, although it has been shown that localization effects, if they appear, would be masked by ballistic effects when the samples are finite. ${ }^{8,9}$

In the present work, we will therefore not address "bulk" samples but finite systems of up to a few micrometers in length. In this way, an important part of the transport is ballistic and scattering effects come in as a resistive correction. Therefore all the aforementioned problems are much less of a concern. Localization and multiple-scattering effects, which may occur mostly in the high frequencies, will not show up in a thermal conductance measurement. Further- 
more, we will restrict ourselves to cryogenic temperatures, at which anharmonic scattering can be quite neglected and the phonons' scattering cross section is mostly isotropic. Thus, in this quasiballistic finite length limit, it is reasonable to employ the total cross section of the individual cluster scatterers to estimate their effect on thermal conductance.

The correct scattering cross section must nonetheless take into account the change in overall density of the medium. The coherent-potential approximation ${ }^{24}$ in the limit $\Delta M$ $\ll M$, which is valid here, yields the following mean-free path of a phonon in branch $i$ with wave vector $\vec{q}$ (Refs. 19 and 25):

$$
\lambda_{i}^{-1}(\vec{q})=x(1-x) \sigma(\vec{q}) / A,
$$

where $x$ is the isotope volume fraction in the sample. We are considering randomly distributed scatterers and we thus neglect interference effects between scatterers.

Below $50 \mathrm{THz}$ the phonon dispersion and scattering cross sections are isotropic to a good approximation. In temperature terms this corresponds to $50 \mathrm{THz} / k_{B} \sim 382{ }^{\circ} \mathrm{K}$, five times larger than the highest temperature we consider here $(77 \mathrm{~K})$. So at $77 \mathrm{~K}$ and below it is reasonable to consider the phonon mean-free path to be isotropic, depending only on the branch and frequency but not on direction. Then, in a sample with perfect contacts and a resistive region of length $L$, in the presence of elastic scattering only, the thermal conductance per unit cross section is approximately ${ }^{26}$

$\sigma_{\text {cond }}=\frac{1}{D} \int_{0}^{\infty} \hbar \omega \frac{d f}{d T} \sum_{i}\left(1+\frac{4}{\pi} \frac{L}{2 \lambda_{i}(\omega)}\right)^{-1} \mathcal{T}_{i}(\omega) d \omega / 2 \pi$,

where $i$ extends to the three acoustic and three optical branches, and $D=3.35 \AA$ is the graphitic interlayer spacing. $\mathcal{T}_{i}(\omega)=\frac{1}{2} \int_{B Z} \delta\left[\omega-\omega_{i}(\vec{q})\right]\left|\frac{d \omega_{i}(\vec{q})}{d q_{x}}\right| d \vec{q} / 2 \pi$ is the ballistic transmission associated with branch $i$, where the integral is performed within the two-dimensional first Brillouin zone. ${ }^{27} \lambda_{i}$ is defined in terms of the scattering cross section for branch $i$ [Eq. (14)]. In the limit $L \rightarrow 0$ Eq. (15) yields the ballistic conductance, the inverse of which is referred to as the intrinsic resistance of the contact. ${ }^{28}$ It is convenient to express the results in terms of an equivalent thermal conductivity that can be compared to that of graphite, $\kappa \equiv L \sigma_{\text {cond }}$.

Figure 5 shows the computed thermal conductivity for different cluster sizes up to 30 atoms. In each case, five values are given corresponding to isotope concentrations (or volume fractions) of $10 \%, 20 \%, 30 \%$, and $40 \%$. At liquid nitrogen temperature $(77 \mathrm{~K})$ an important effect due to clustering is noticeable already for a sample $2.5 \mu \mathrm{m}$ long, leading to an additional $\sim 450 \mathrm{~W} / \mathrm{mK}$ reduction in thermal conductivity with respect to the independent scatterer case. At $10 \mathrm{~K}$, a much longer sample of $30 \mu \mathrm{m}$ is needed to observe a similar effect since the low-frequency phonons active at this temperature have very long mean-free paths. If ${ }^{14} \mathrm{C}$ isotopes are used instead, effects can be up to four times stronger. Graphite samples are expected to display qualitatively similar clustering effects as those shown in graphene. The latter has been chosen here for computational simplicity but graphite might perhaps be a simpler experimental candidate for the verification of these effects.

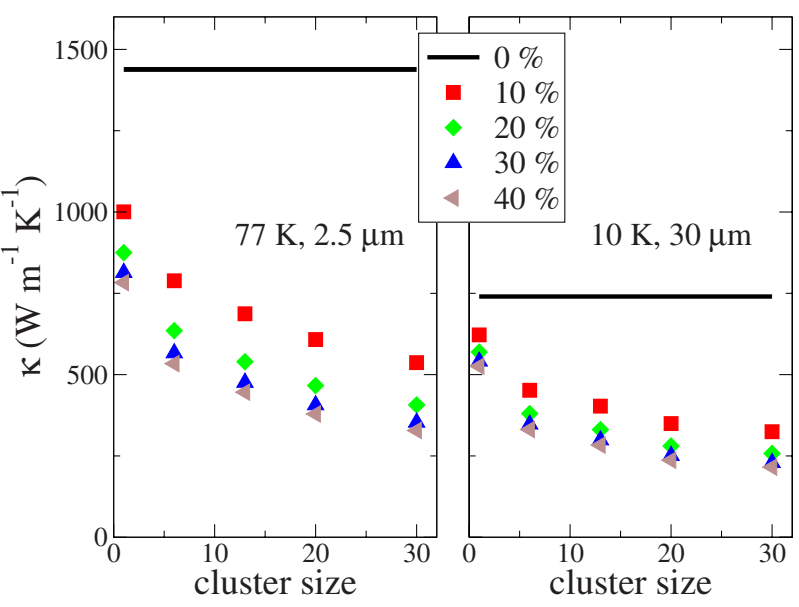

FIG. 5. (Color online) Thermal conductivity versus number of atoms in cluster for a $2.5-\mu \mathrm{m}$-long sample at a $77 \mathrm{~K}$ (filled symbols) and for a $30-\mu \mathrm{m}$-long sample at $10 \mathrm{~K}$ (empty symbols) for different ${ }^{13} \mathrm{C}$ isotopic concentrations.

The thermal-conductivity reduction depends on cluster size more weakly than $N^{2}$, mainly due to the anomalous dependence of the out of plane branch cross section with $N$ in the midfrequency range, discussed earlier. If increasingly larger clusters are considered while keeping a constant overall isotope concentration, one can expect further reduction in the thermal conductivity down to a minimum value for a given cluster size. For even larger clusters, the cross section will eventually become equal to the geometric limit at virtually all frequencies, thus scaling like $\sqrt{N}$. When this is the case, the inverse mean-free path given by Eq. (14) goes as $\lambda^{-1} \propto 1 / \sqrt{N}$ (because $A \propto N$ ) and so the thermal conductivity will start increasing rather than decreasing with $N$. A similar minimum has been predicted for three-dimensional nanoparticles in bulk materials. ${ }^{5}$ Determining the large cluster sizes necessary to reach such minimum is a very computationally demanding problem beyond the scope of this paper.

\section{A suggested route toward synthesizing isotopic-cluster graphene}

"Isotope clusters" might at first sight seem like an academic problem. Isotopes do not chemically differ among themselves so they do not naturally tend to form clusters. ${ }^{29}$ Thus one might think that their distribution in a crystal will always be random. It may nonetheless be conceivable to grow graphite and graphene, containing isotope aggregates of regular size. A suggestion on how this could be achieved is by chemical vapor deposition of hydrocarbons. Two different sources of the same hydrocarbon but containing, respectively, pure ${ }^{12} \mathrm{C}$ and ${ }^{13} \mathrm{C}$ or ${ }^{14} \mathrm{C}$, would be simultaneously deposited to fully cover a single-crystal metal surface (such as $\mathrm{Pt}$ ). Subsequent annealing results in the dehydrogenation of the adsorbed molecules and graphitization of the carbon atoms covering the metal. The obtained graphite layers might contain isotope clusters with roughly the number of carbon atoms in the precursor molecules. This technique for producing thin graphite layers is well known ${ }^{30}$ but we are not aware of any attempts at producing isotope clusters in the way we have depicted. 
An alternative and perhaps more promising approach can also be suggested, replacing the hydrocarbons by $\mathrm{C}_{60}$ fullerenes. The fullerene approach has been successfully applied to produce controlled isotopic domains in carbon nanotubes, where it was shown that the isotopes remain pinned to the original location of the fullerenes. ${ }^{31,32}$ In the case of carbon nanotubes, important effects of clustering on the thermal conductivity have been theoretically predicted. ${ }^{10}$

It might then be possible to mix two isotopic sources of fullerenes similarly as described above, to produce graphitic structures with isotopic clusters. Graphitization in this way has been recently demonstrated. ${ }^{33}$ Graphene can be subsequently isolated via known techniques. It has already been demonstrated that micrometer long graphene sheets can be suspended to measure their thermal conductivity. ${ }^{34}$ The same approach may be used to characterize the thermal conductivity of the isotopic-cluster graphene samples just described.

\section{CONCLUSIONS}

An $a b$ initio atomistic calculation of the scattering cross section of different clusters was presented, showing consid- erable structure and various features qualitatively different from the predictions of analytical approaches previously employed in the literature. Clustering is found to lead to a very marked observable reduction in the thermal conductivity of graphene, well below the disordered isotope limit. Due to the chemically inactive character of isotopic impurities, experiments on isotopic-cluster graphene like the ones proposed here might be useful in deepening our understanding of phonon behavior at the nanoscale.

Note added in proof. Graphene with defined isotopic domains has been recently synthesized. ${ }^{37}$

\section{ACKNOWLEDGMENTS}

K.E. thanks the Center for Computational Materials Science of IMR at Tohoku University for generous supercomputer time. N.M. thanks F. Mauri and G. Stoltz for discussions. We thank Marcel Mohr for providing the experimental data in Fig. 1. This project was supported by NSF under Grants No. 0651310, No. 0651427, and No. 0651381, and by ANR.
${ }^{1}$ L. A. Turk and P. G. Klemens, Phys. Rev. B 9, 4422 (1974).

${ }^{2}$ G. A. Slack and M. A. Hussain, J. Appl. Phys. 70, 2694 (1991).

${ }^{3}$ J. M. Ziman, Electrons and Phonons (Oxford University Press, New York, 2001).

${ }^{4}$ W. Kim, J. Zide, A. Gossard, D. Klenov, S. Stemmer, A. Shakouri, and A. Majumdar, Phys. Rev. Lett. 96, 045901 (2006).

${ }^{5}$ N. Mingo, D. Hauser, N. P. Kobayashi, M. Plissonnier, and A. Shakouri, Nano Lett. 9, 711 (2009).

${ }^{6}$ A. Majumdar, J. Heat Transfer 115, 7 (1993).

${ }^{7}$ W. Kim and A. Majumdar, J. Appl. Phys. 99, 084306 (2006).

${ }^{8}$ I. Savic, N. Mingo, and D. A. Stewart, Phys. Rev. Lett. 101, 165502 (2008).

${ }^{9}$ G. Stoltz, M. Lazzeri, and F. Mauri, J. Phys.: Condens. Matter 21, 245302 (2009).

${ }^{10}$ G. Stoltz, N. Mingo, and F. Mauri, Phys. Rev. B 80, 113408 (2009).

${ }^{11}$ A. A. Maradudin, E. W. Montroll, G. H. Weiss, and I. P. Ipatova, Theory of Lattice Dynamics in the Harmonic Approximation, Solid State Physics, 2nd ed. (Academic, New York, 1971).

${ }^{12}$ E. N. Economou, Green's Functions in Quantum Physics (Springer, Berlin, 1983).

${ }^{13}$ N. Mingo and L. Yang, Phys. Rev. B 68, 245406 (2003).

${ }^{14}$ G. Kresse and J. Furthmuller, Phys. Rev. B 54, 11169 (1996); Comput. Mater. Sci. 6, 15 (1996).

${ }^{15}$ K. Esfarjani and H. T. Stokes, Phys. Rev. B 77, 144112 (2008).

${ }^{16}$ M. Mohr, J. Maultzsch, E. Dobardzic, S. Reich, I. Milosevic, M. Damnjanovic, A. Bosak, M. Krisch, and C. Thomsen, Phys. Rev. B 76, 035439 (2007).

${ }^{17}$ L. Wirtz and A. Rubio, Solid State Commun. 131, 141 (2004).

${ }^{18}$ N. Mounet and N. Marzari, Phys. Rev. B 71, 205214 (2005).

${ }^{19}$ H. Ehrenreich and L. M. Schwartz, in Solid State Physics, edited by H. Ehrenreich, F. Seitz, and D. Turnbull (Academic, New York, 1976), Vol. 31, p. 149.

${ }^{20}$ R. Haydock, V. Heine, and M. J. Kelly, J. Phys. C 5, 2845 (1972).

${ }^{21}$ W. Zhang, N. Mingo, and T. S. Fisher, Numer. Heat Transfer,
Part A 51, 697 (2007).

${ }^{22}$ W. Zhang, N. Mingo, and T. S. Fisher, J. Heat Transfer 129, 483 (2007).

${ }^{23}$ F. Guinea, C. Tejedor, F. Flores, and E. Louis, Phys. Rev. B 28, 4397 (1983).

${ }^{24}$ Ping Sheng, Introduction to Wave Scattering, Localization and Mesoscopic Phenomena (Springer, New York, 2006).

${ }^{25}$ B. Abeles, Phys. Rev. 131, 1906 (1963).

${ }^{26}$ N. Mingo and D. A. Broido, Nano Lett. 5, 1221 (2005).

${ }^{27}$ N. Mingo and D. A. Broido, Phys. Rev. Lett. 95, 096105 (2005).

${ }^{28}$ S. Datta, Electronic Transport in Mesoscopic Systems (Cambridge University Press, Cambridge, England, 1995).

${ }^{29}$ This statement is not strictly true. Some older theoretical work has pointed at the existence of thermodynamically driven interactions between isotopes at very low temperature (see Refs. 11, 35, and 36). Nevertheless, the small mass differences between carbon isotopes suggest that this may not be a relevant effect in the current case.

${ }^{30}$ T. A. Land, T. Michely, R. J. Behm, J. C. Hemminger, and G. Comsa, Surf. Sci. 264, 261 (1992).

${ }^{31}$ V. Zólyomi, F. Simon, Á. Rusznyák, R. Pfeiffer, H. Peterlik, H. Kuzmany, and J. Kürti, Phys. Rev. B 75, 195419 (2007).

${ }^{32}$ F. Simon, C. Kramberger, R. Pfeiffer, H. Kuzmany, V. Zólyomi, J. Kürti, P. M. Singer, and H. Alloul, Phys. Rev. Lett. 95, 017401 (2005).

${ }^{33}$ H. Lim, H. S. Shin, H. J. Song, and H. C. Choi, Nanotechnology 20, 145601 (2009).

${ }^{34}$ A. A. Balandin, S. Ghosh, W. Bao, I. Calizo, D. Teweldebrhan, F. Miao, and C. N. Lau, Nano Lett. 8, 902 (2008); S. Ghosh, I. Calizo, D. Teweldebrhan, E. P. Pokatilov, D. L. Nika, A. A. Balandin, W. Bao, F. Miao, and C. N. Lau, Appl. Phys. Lett. 92, 151911 (2008)

${ }^{35}$ E. Montroll and R. B. Potts, Phys. Rev. 100, 525 (1955).

${ }^{36}$ E. Montroll and R. B. Potts, Phys. Rev. 102, 72 (1956).

${ }^{37}$ X. Li, W. Cai, L. Colombo, and R. S. Ruoff, Nano Lett. 9, 4268 (2009) 